\title{
Acoustic Imaging of Aircraft Wake Vortex Dynamics
}

\author{
Frank Y. Wang ${ }^{*}$, Hadi S. Wassaf ${ }^{\dagger}$ and Andrew Gulsrud ${ }^{*}$ \\ USDOT John A. Volpe National Transportation Systems Center, Cambridge, MA 02142 \\ Donald P. Delisi ${ }^{\S}$ \\ NorthWest Research Associates, Inc., Bellevue, WA 98007 \\ and \\ Robert P. Rudis* \\ Aviation Turbulence Research, Burlington, MA 01803
}

\begin{abstract}
The experience in utilizing a phased microphone array to passively image aircraft wake vortices is highlighted. It is demonstrated that the array can provide visualization of wake dynamics similar to smoke release or natural condensation of vortices. Examples on how the technique has been integrated with other data to address some of the research issues in wake vortices are documented. These topics include the initial vortex spacing characterization, collaboration with simultaneous pulsed Lidar data to provide a more complete understanding of vortices at a late stage in their evolution, and correlation between atmospheric turbulence with the time-scale involved for vortices developing into a very contorted state. In addition, the paper provides a comparison between the vortex tracks as inferred from their acoustics characteristics vs. those detected from the velocity field (i.e., pulsed Lidar). A preliminary attempt is also made to examine the global link between wake vortex sound and vortex circulation, suggesting that there could be a power law relationship between the two datasets.
\end{abstract}

\section{Introduction}

A IRCRAFT wake vortex evolution has been a subject of both practical and academic interest since the 1970s. This paper reports highlights of the recent experience in using a phased microphone array to passively detect and track aircraft vortices in the out-of-ground effect (OGE) region (loosely defined here as vortex altitudes higher than the aircraft wingspan). The concept of using the sound vortices generate as the basis of a wake sensor, to our knowledge, was first discussed in the 1970s. ${ }^{1,2}$ However, the passive wake acoustics concept was not actively pursued until recent years in the United States under the program SOCRATES ${ }^{3}$ (Sensor for Optically Characterizing Remote Atmospheric Turbulence Emanating Sound), which is an opto-acoustic sensor array under development by Flight Safety Technologies (FST) and Lockheed Martin Corporation (LM). Independent of the SOCRATES effort, the C-Wake program of the European Commission recently engaged the German Aerospace Research Center in Berlin (DLR-Berlin) to explore the utility of a phased microphone array ${ }^{4}$ to investigate whether the noise signatures of wake vortices contain valuable aerodynamic information such as turbulence and/or hazard.

In order to better understand the fundamental phenomenology of wake vortex sound, from August to September 2003, under the sponsorship of the National Aeronautics and Space Administration (NASA), the United States Department of Transportation John A. Volpe National Transportation Systems Center (USDOT Volpe Center) and its supporting contractors conducted an extensive field measurement campaign to characterize the acoustic signature of OGE aircraft wake vortices. A large, both spatially as well as in number of elements, phased microphone array was fielded at Denver International Airport as the central part of this effort. In addition, a suite of aerodynamic ground-truth and meteorological sensors were fielded to support the analyses of the wake acoustics data.

The Denver microphone test also provided an opportunity to conduct field evaluation of recent modifications to the laser-based wake acoustic sensor SOCRATES. A four-beam SOCRATES sub-system was also fielded by FST-LM at

\footnotetext{
Aerospace Engineer, Advanced CNS Technologies Division, Senior Member AIAA.

Electrical Engineer, Advanced CNS Technologies Division.

Aerospace Engineer, Advanced CNS Technologies Division.

$\S$ Senior Research Scientist and Chairman of the Board, Senior Member AIAA.

** Engineering Manager.
} 
the Denver test site. In addition, under the FST funding, DLR-Berlin deployed its phased microphone array to further characterize the acoustic properties of aircraft wake vortices. Although the Denver experiment presents ample opportunities for cross verification of sensor performances and processing techniques, the present paper will only focus on reporting the results from the NASA-USDOT phased microphone array, their comparison to the pulsed Lidar data and their correlation with ambient turbulence. It is important to note that analyses of the wake acoustics data from the Denver 2003 test are being carried out at a number of organizations including NASA Langley Research Center, USDOT Volpe Center, OptiNav, Inc., Florida Atlantic University, Flight Safety Technologies, Lockheed Martin Corporation and DLR-Berlin. Preliminary results from the Denver 2003 experiment have been presented in Refs. 5-6. Additional results are also found in Refs. 7-9. The present paper, along with Ref. 10, highlight the recent results from the USDOT Volpe Center group and represent a fractional contribution to the overall wake acoustics effort undertaken by the consortium. The present study, however, distinguishes itself from other passive wake acoustics investigations to date by focusing on utilizing the phased microphone results to address fundamental wake vortex issues.

\section{Test Description}

Phased microphone array measurements were conducted at Denver International Airport at a location under the flight path of runway 16L, two miles from the runway threshold where the nominal arriving aircraft altitude was approximately 700 feet above ground level. Arrival aircraft are the focus at this time in part because the competing jet noise is greatly reduced in such a flight profile. The selection of the Denver International Airport is motivated both by its diverse aircraft mix, as well as the pristine acoustic environment.

Fig. 1 shows an aerial photograph of the test site. The magnetic-north direction in Fig. 1 is approximately pointing at the 11 o'clock direction. In the present paper, only the most pertinent information on sensors utilized in the present paper is briefly described in the paragraphs to follow. Additional information on the overall instrumentation suite can be found in Refs. 5-6.

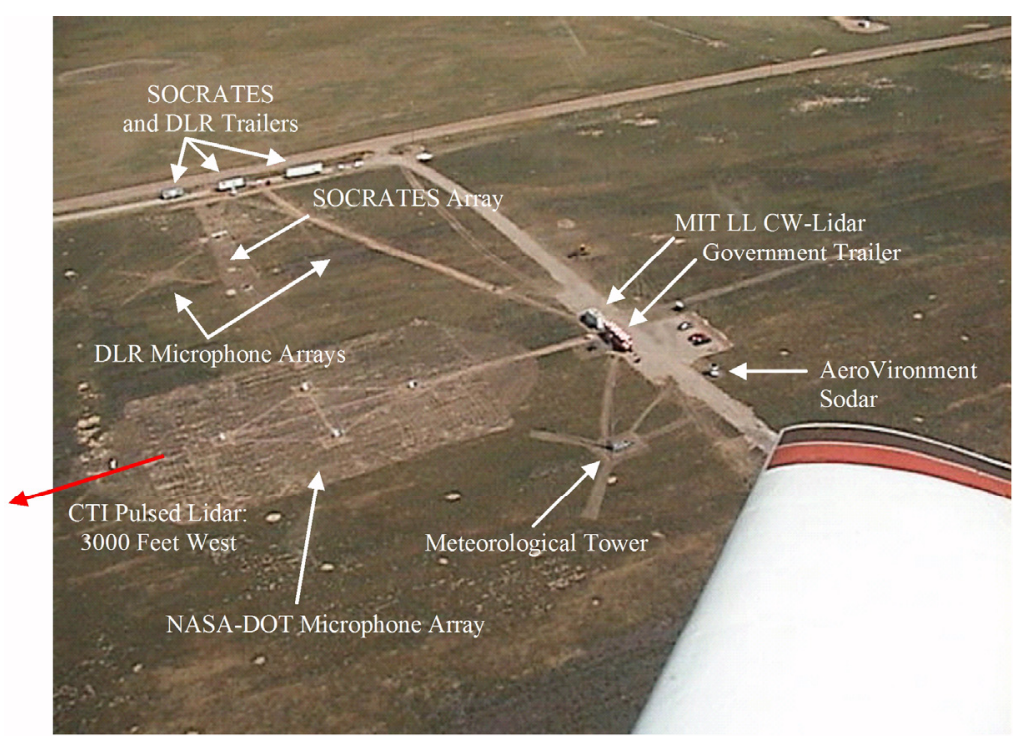

Figure 1. Aerial Photograph of the Test Site Illustrating the Relative Locations of Various Sensors.

\section{A. Phased Microphone Array}

The array was designed by OptiNav, Inc., with hardware integration by both Microstar Laboratories, Inc. and OptiNav, Inc. The array comprised of 252 electret microphones and arranged along multiple curved lines with a logarithmic spacing of elements. The array pattern is shown in Fig. 2. The physical dimensions of the array deployed were 398 feet in the direction perpendicular to the flight path (roughly the east-west direction) and 102 feet along the direction of flight (roughly the north-south direction). The designed frequency of the array was from $20-1000 \mathrm{~Hz}$. for the investigation. Additional details on the microphone array design and associated components can be found in Ref. 6 . 


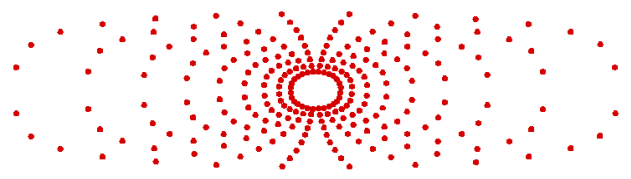

Figure 2. The Array Pattern.

\section{B. Pulsed Lidar}

A pulsed Doppler Lidar (Light Detection and Ranging) from Coherent Technologies, Inc. (CTI) was deployed as one of the ground-truth wake vortex tracking sensors. The system was located 3000 feet west of the microphone array site due to the need of a minimum standoff distance as well as topographical considerations. It operates at 2 micron wavelength, pulsing at a rate of $500 \mathrm{~Hz}$ and continuously scanning in an arc motion to obtain line-of-sight velocity measurements by detecting the Doppler shift of the light backscattered from aerosols naturally present in the atmosphere. Like pulsed radio frequency radars, the Lidar measures distance by range-gating the returned signal. Circulation estimates from the pulsed Lidar are derived from a two dimensional match filter using a dispersion vortex model. Additional information about the pulsed Lidar can be found in Refs. 11-12. A photograph of the pulsed Lidar is shown in Fig. 3.

\section{Sonic Anemometer}

A Metek ultrasonic anemometer was introduced to the test approximately half way into the Denver 2003 wake acoustics test and provided the opportunity to characterize the turbulent characteristics at the test area, at least near the surface. By measuring the traveling times of ultrasonic pulses between three pairs of alternating transmitters and receivers, three component velocities and virtual temperature at the rate of $10 \mathrm{~Hz}$ were obtained. The sensor was mounted on a $24 \mathrm{ft}$ pole above the ground, supported by the side-edge of the instrumentation trailer. The mounting configuration was selected to optimize measurements during head-wind conditions, which is the preferred operational mode of landing aircraft. In relation to the aircraft altitude, the sonic anemometer height represents approximately 7 wingspan distances from the glide slope altitude for Large weight turbulence class aircraft, and less for Heavy weight class aircraft. A photograph of the sonic anemometer is shown in Fig. 4.

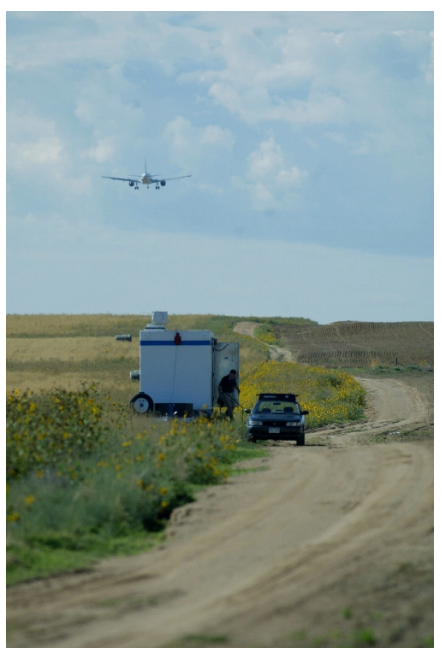

Figure 3. The CTI Pulsed Lidar.

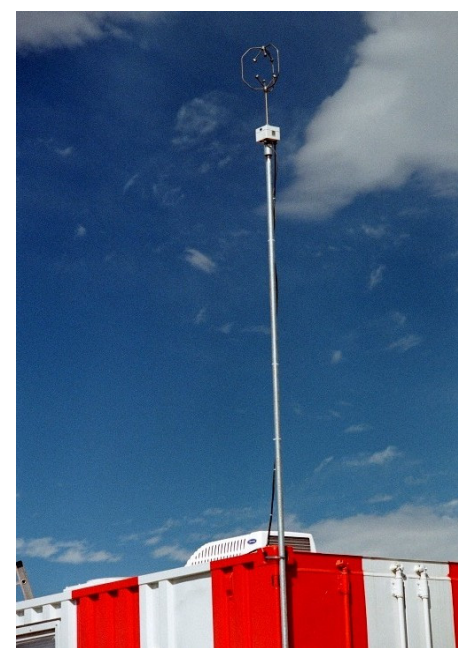

Figure 4. The Metek Sonic Anemometer.

\section{Phased Microphone Array Dataset}

The period of September 3rd to September 19th, 2003 represents the time during which the measurement equipment became fully online. The phased array measurement dataset during that time consisted of 1011 flyovers. After excluding files with late or false triggering, departures and files that are too short in duration to be meaningful, a total of 771 measurements associated with arrivals form the dataset of the phased microphone array test. In terms of weight, the aircraft inventory in the dataset ranged from Beech-1900 up to Boeing-777. However, as comparisons and correlations 
are made with either the pulsed Lidar or sonic anemometer, or performing a different kind of acoustic analysis (to be discussed throughout the paper), the associated database size for those purposes will decrease.

\section{Results and Discussion}

\section{A. Source Localization Maps by Beamforming on a Horizontal Plane}

The first stage of the data processing aimed at providing a global and baseline description of the data. This is accomplished by performing beamforming analyses at two frequency bins, e.g., 0-200 and 200-400 Hz, respectively. Subsequent spectral characterization of wake vortices confirmed that $400 \mathrm{~Hz}$ is a reasonable upper bound, as there is little acoustic energy above $400 \mathrm{~Hz}$. For these analyses, delay-and-sum beamforming in the time domain was performed at a focusing horizontal plane 500 feet above the ground to provide evidence of wake noise detection via source localization maps. The essence of the source localization map and important considerations in interpreting the results are documented in Ref. 6 . The beamforming region for analyses of this type is $1500 \mathrm{ft}$ in the east-west direction and 1000 feet in the north-south direction. Also for these "first iteration coarse grid" studies, a beamforming grid resolution of $20 \mathrm{ft}$ by $20 \mathrm{ft}$ was employed. It is important to note that the north-south extent of the beamforming region corresponds to approximately one wavelength of Crow instability for Large class aircraft. ${ }^{13}$ This particular feature of the data processing will be discussed further subsequently. On selected tracks of special interest, higher spatial resolution beamforming was also performed using frequency bins of either $0-300 \mathrm{~Hz}^{6}$ with $5 \mathrm{ft}$ by $5 \mathrm{ft}$ grid or 200-400 $\mathrm{Hz}$ using $10 \mathrm{ft}$ by $10 \mathrm{ft}$ resolution with sub-grid interpolation. In all cases, source localization maps were made by integrating the acoustic power over 2 seconds at each grid point. Finally, a simulation of the array response in the vertical direction at various frequencies of interest shows that a fixed beamforming altitude of 500 feet is more than adequate for analyses of this type. The poor resolution of the array in the vertical direction permits a constant altitude beamforming such that vortex sound, when emitted with sufficient loudness, can be revealed from aircraft altitude down to approximately 250 feet above the ground.

An example of the higher resolution beamforming run at $200-400 \mathrm{~Hz}$ is shown in Fig. 5. The particular flyover was a B735 run on September 16th, 2003. In the sequence of images shown in Fig. 5, the aircraft enters the beamforming space from north to south (or from the upper edge of the frame to the lower edge of the frame). When the aircraft is shown in the middle of the frame, it is denoted as occurring at $\mathrm{t}=0 \mathrm{sec}$. For the series of images shown in Fig. 5, the source localization map for the flyover frame is omitted. Instead, the sequence of images starts at two seconds after flyover, which is indicated as $\mathrm{t}=02 \mathrm{sec}$. in the first frame. Lastly, a scale of 0.4 to $5 \mathrm{~dB}$ was used to color the noise source contour. The bright spot at the lower edge of the $\mathrm{t}=02 \mathrm{sec}$. frame represents the aircraft noise. A pair of horizontal columnar noise sources trailing behind the aircraft is evident for at least 12 seconds after the aircraft left the beamforming space. The spacing between the two columnar noise sources is within the expected range of vortex pair separation distance (see Ref. 6, and this point will also be further discussed later). The wake vortex noise in the columnar form disappeared 30 seconds after the flyover in this particular example. A period of silence in the beamforming space is then observed, followed by the appearance of a ring structure drifting from the south to north direction. Recall that the length of the beamforming space in the north-south direction is approximately that of the wavelength of Crow instability. ${ }^{13}$ In this particular example, it is seen that after 30 seconds of flyover, the vortex developed into a Crow instability state and the beamforming region happens to be in the space between two successive vortex rings. As the headwind associated with this landing blows the vortex ring into the beamforming region, the ring like structure is then acoustically imaged.

The series of images in Fig. 5 revealed a number of important findings. Firstly, it is seen that the phased microphone array technique has enough resolution to discern the initial vortex spacing. The spacing between vortices at the initial state has modeling application and at a later stage, the longitudinal variation of the vortex location contains encounter hazard information. Also, although vortex oval size is up to twice the aircraft wingspan, the source localization maps reveal that the vortex sound generation is predominantly from a confined region either in or around the cores. More fundamentally, acoustic imaging of the vortex noise allows visualization of the wake evolution in a manner similar to observing smoke release in dedicated flight tests, or from vortex-induced condensation marking the vortex cores on very moist days. It is apparent that these acoustic studies can offer the unique opportunity to visualize aspects of the wake vortex dynamics that would otherwise not be possible in the typical airport environment. Additional analysis (to be presented later) by comparing the evolution of these noise sources with pulsed Lidar measurements will provide further confidence that wake vortices in fact have been acoustically imaged from the Denver 2003 phased microphone array data. Source localization maps of this type will subsequently be utilized to compile statistics of wake vortex sound detection and perform visualization studies of wake vortex dynamics. 


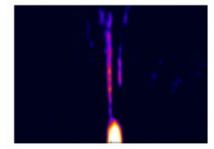

$\mathrm{t}=02 \mathrm{sec}$.

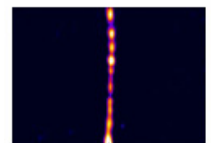

$\mathrm{t}=18 \mathrm{sec}$.

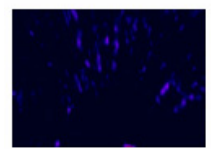

$t=34 \mathrm{sec}$.

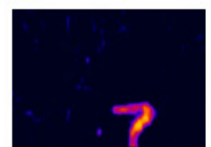

$t=50 \mathrm{sec}$.

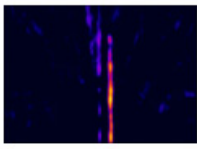

$\mathrm{t}=04 \mathrm{sec}$.

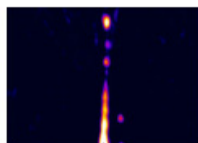

$\mathrm{t}=20 \mathrm{sec}$.

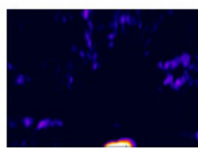

$t=36 \mathrm{sec}$

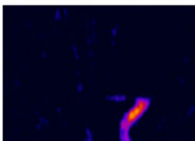

$t=52 \mathrm{sec}$

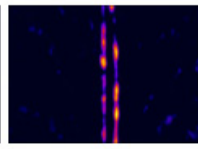

$\mathrm{t}=06 \mathrm{sec}$.

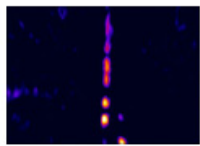

$\mathrm{t}=22 \mathrm{sec}$.

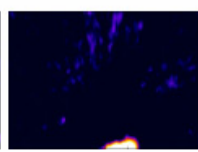

$t=38 \mathrm{sec}$.

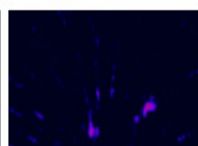

$\mathrm{t}=54 \mathrm{sec}$

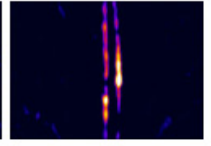

$\mathrm{t}=08 \mathrm{sec}$.

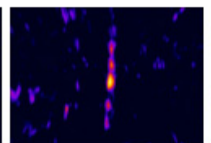

$\mathrm{t}=24 \mathrm{sec}$.

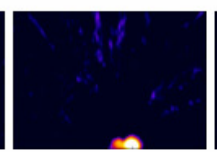

$t=40 \mathrm{sec}$.

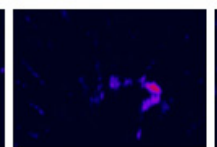

$t=56 \mathrm{sec}$.

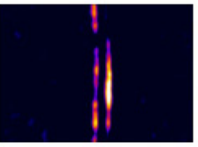

$\mathrm{t}=10 \mathrm{sec}$.

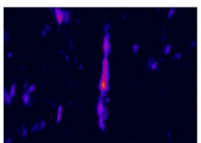

$\mathrm{t}=26 \mathrm{sec}$

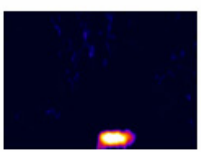

$t=42 \mathrm{sec}$

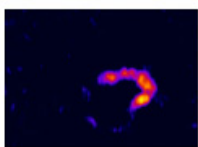

$\mathrm{t}=58 \mathrm{sec}$.

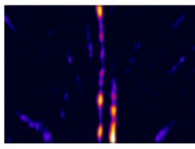

$\mathrm{t}=12 \mathrm{sec}$.

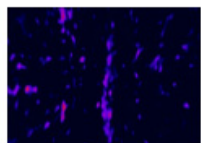

$\mathrm{t}=28 \mathrm{sec}$.

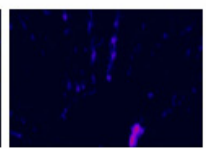

$t=44 \mathrm{sec}$

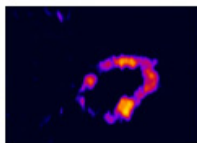

$\mathrm{t}=60 \mathrm{sec}$

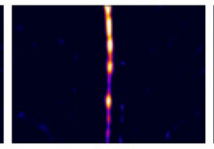

$\mathrm{t}=14 \mathrm{sec}$.

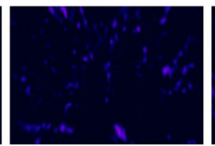

$t=30 \mathrm{sec}$.

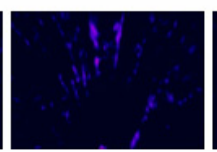

$t=46 \mathrm{sec}$.

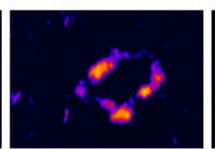

$\mathrm{t}=62 \mathrm{sec}$.

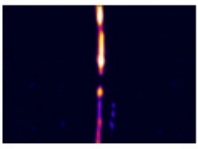

$\mathrm{t}=16 \mathrm{sec}$.

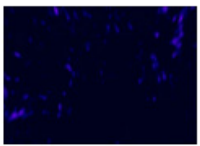

$t=32 \mathrm{sec}$.

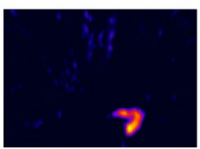

$t=48 \mathrm{sec}$.

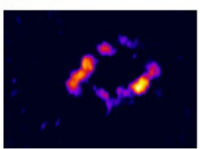

$\mathrm{t}=64 \mathrm{sec}$.

Figure 5. A B735 Arrival on September 16, 2003. The beamforming altitude was 500 feet AGL and the frequency range was from $200-400 \mathrm{~Hz}$

Fig. 6 summarizes the statistics of vortex noise detection as revealed by the source localization maps and shows the level of wake noise consistency examined by aircraft type in the two frequency bins (i.e., 0-200 and 200-400 Hz). It is interesting to note that small turboprop aircraft generate detectable wake noise, even though the sample size is small and detection rate is not high. It is also surprising to see that a significant portion of the Airbus 318-320 series aircraft do not produce detectable sound in their wake vortices in the design frequency of the array. The relatively low percentage of wake noise detection in 200-400 Hz associated with Boeing 772 is also surprising, but the associated database is not very large. However, the same aircraft produced consistent wake sound in the lower frequency range. The second heaviest aircraft in the database is Airbus 343, and the small data sample there also showed poor sound detection rate. It is known that every single aircraft generates a wake but it is not clear from the data if every wake generates a detectable wake sound. A preliminary examination of the flyby cases that resulted in no detectable wake sound included looking at possible influence of the meteorological conditions, such as wind magnitude, atmospheric stability and turbulence. However, no meaningful correlation was found between meteorological condition and the absence of an acoustic wake signal. It is however noted that the poor aircraft wake noise generators tend to be, but not always, newer aircraft, hence presumably with aerodynamically cleaner wings. In the case of some of the Airbus models, the additional physical attribute of the poor wake noise makers is the relatively simple flap design. Although the "silent vortex" cases represent in some sense, the most interesting and important cases to study from a sensor development perspective, analyses to date have mostly been performed on cases that do produce detectable vortex sound. The cases of the "silent vortex" will form the basis of a future study. 


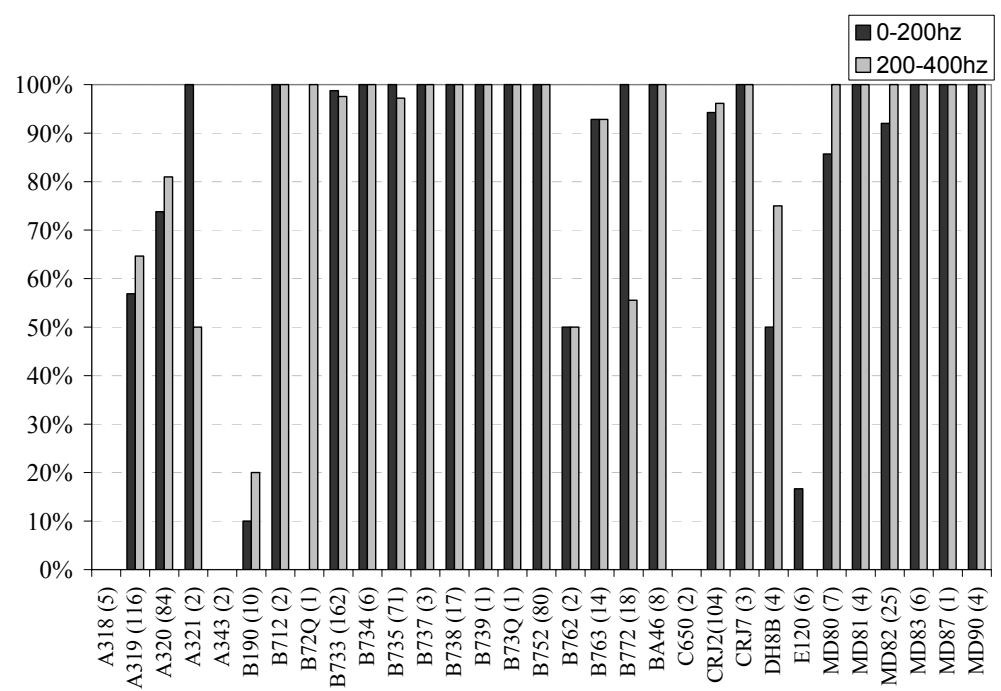

Figure 6. Statistical Compilation of the Wake Vortex Noise by Aircraft Model.

The Number Aircraft in the Statistics is Indicated in Parenthesis After the Aircraft Model.

\section{B. Initial Vortex Separation Distance}

Beamforming in the vertical plane directly above the array center was also performed to support additional studies. The wake sound evolution produced in this manner (i.e., lateral and vertical wake positions in the plane above the array as a function of time) allows direct comparison with pulsed Lidar measurements (where the Lidar scans the wake in a plane perpendicular to the flight path) as well as obtaining the initial vortex spacing as inferred by the vortex sound. Note that extraction of the vortex separation distance from vertical beamforming avoids the parallax issue associated with horizontal beamforming. Vertical beamforming was performed in the range of $0-300 \mathrm{~Hz}$ and 461 of these tracks were analyzed. The beamforming space covers 1500 feet in the east-west direction and 900 feet (from 100 to $1000 \mathrm{ft}$ AGL) vertically. The grid resolutions in the lateral and vertical directions were $20 \mathrm{ft}$ and $25 \mathrm{ft}$ respectively, with subgrid interpolation to enhance the effective resolution. The integration time was also selected to be 2 seconds. The generation of the vertical beamforming tracks is similar to that of Ref. 6, but differs in the treatment of array shading, grid resolution and image processing details. ${ }^{10}$

Results of the initial vortex separation distance (most commonly referred to as $b_{o}$ ) are presented in Fig. 7 for two predominant aircraft types in the vertical beamforming database, namely Boeing 737s (excluding the 800 and 900 series) and Boeing 757s.
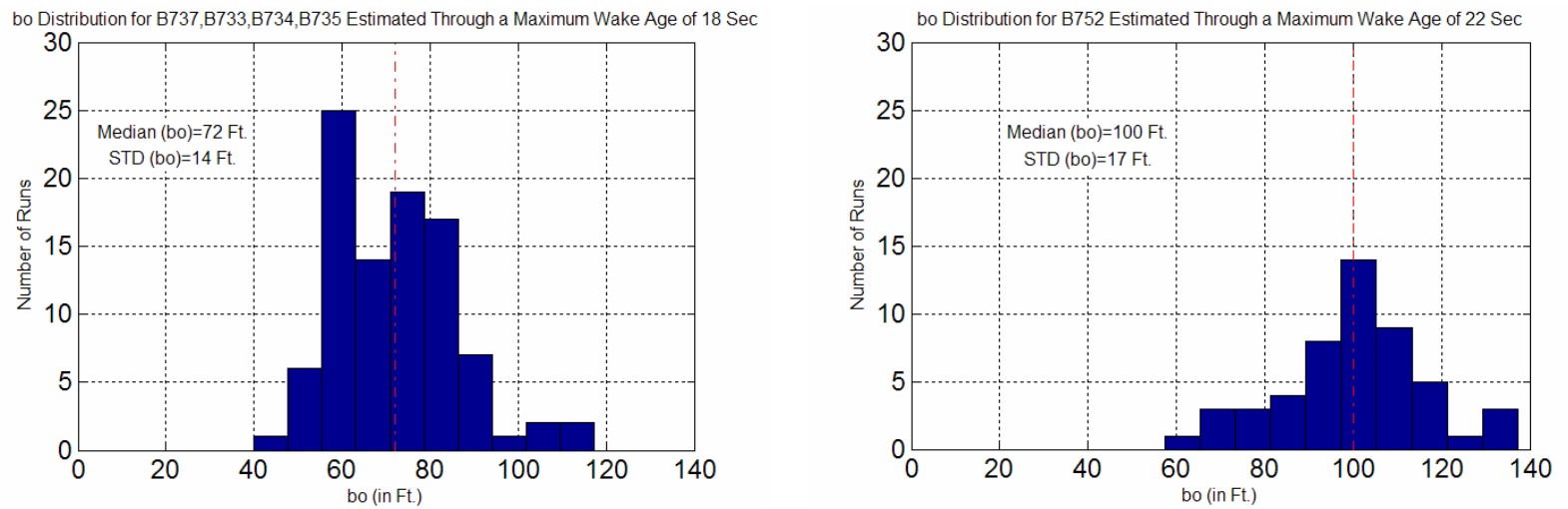

Figure 7. Distribution of the Initial Vortex Separation Distance.

The $b_{o}$ values were taken from the median age of the wakes up to $1.5 T$, where $T$ is the customary non-dimensional time in wake vortex studies ${ }^{15}$ (i.e., $T$ is the theoretical time for a vortex pair to descend a distance of one $b_{o}$ ). The theoretical values of $b_{o}$ for B737 and B757 aircraft are $75 \mathrm{ft}$ and $98 \mathrm{ft}$, respectively, based on the assumption of a clean wing with elliptical loading. Note that the median values of the measured $b_{o}$ for B737 and B757 are $72 \mathrm{ft}$ and $100 \mathrm{ft}$, 
respectively, which compare very favorably to the theoretical values. It is worth noting that $b_{o}$ in the OGE region is not easily revealed by field measurements of wake vortices. This difficulty is, in part, due to the fact that field measurements usually invoke traditional meteorological sensors, which are not always optimized for characterizing small-scale structures, such as wake vortices. Similarly, aircraft wake vortices are typically too large for traditional fluid mechanics laboratory diagnostic tools to measure.

\section{Comparison with Pulsed Lidar}

One of the research opportunities using the microphone array results is the comparison with the pulsed Lidar data. There are 361 comparison opportunities between horizontal beamforming results and Lidar, and 233 if tracks generated from array vertical beamforming are considered only. A specific example of such a comparison, and the insights that may be gained by merging data from the two sensors, will first be provided, followed by a global statistical comparison of the entire common dataset.

Fig. 8 shows the wake tracks of a B752 as detected by vertical beamforming of the array data and from the pulsed Lidar. The blue and red lines represent the microphone and pulsed Lidar tracks, respectively. Also, note that the array has a two second revisiting time whereas the Lidar acquires track information on a five second interval. The top half of Fig. 8 shows the lateral motion of the wake vs. time, with zero position being the extended runway centerline and positive values indicating the eastward direction. The lateral position trend shows that an easterly wind was present during data collection. The bottom half of Fig. 8 shows the vortex height as a function of time, with altitude indicated in feet above local ground level. The microphone data are usually collected up to 90 seconds, with the microphones activated as the aircraft approaches the array. ${ }^{6}$ Some of the vortex tracks produced from the array are, thus, artificially shortened, as is believed to be the case here. It is also important to keep in mind that the time alignment for the two datasets is not exact, as can be seen in Fig. 8. In general, the lateral position comparison between the two sensors is better than the vertical position comparison.

The horizontal beamforming source localization map for the landing shown in Fig. 8 suggests that vortex linking occurs at around 54 seconds after flyover. Fig. 8 shows that there is a vortex pinch/link-like behavior in the Lidar track which first occurs at about 50 seconds. Likewise, the microphone array lateral position track reveals a similar linking feature somewhere between 2 to 4 seconds later. Thus, the source localization map, showing measurements in a horizontal plane, reveals vortex linking which is similar to an interpretation of Lidar data, which was obtained in a vertical plane. It is known that vortex evolution in a vertical, sheared wind may produce data similar to that in Fig. 8. However, because of the horizontal beamforming, the Lidar data can be interpreted as vortex linking by correlating measurements from these two sensor systems. This example shows that vortex linking is detected and confirmed by using data from both the array and Lidar. The vertical beamforming results showed that two strong noise sources emerged again at 64 seconds after flyover in the general area of where the vortices are anticipated. However, the array data collection unfortunately ended at 66 seconds. Since vortex rings are anticipated after the linking state, it is believed that the Lidar data after the termination of the array data period represent measurements in the ring vortex. It is also interesting to note that there is a change in the descent velocity of the vortex structure at the post-linking stage as seen in the Lidar data. It is also noted that, using the time for the vortices to link as well as the associated change in the vortex height trajectory, the Lidar data may have been misaligned with the array data by approximately 2 to 4 seconds. 

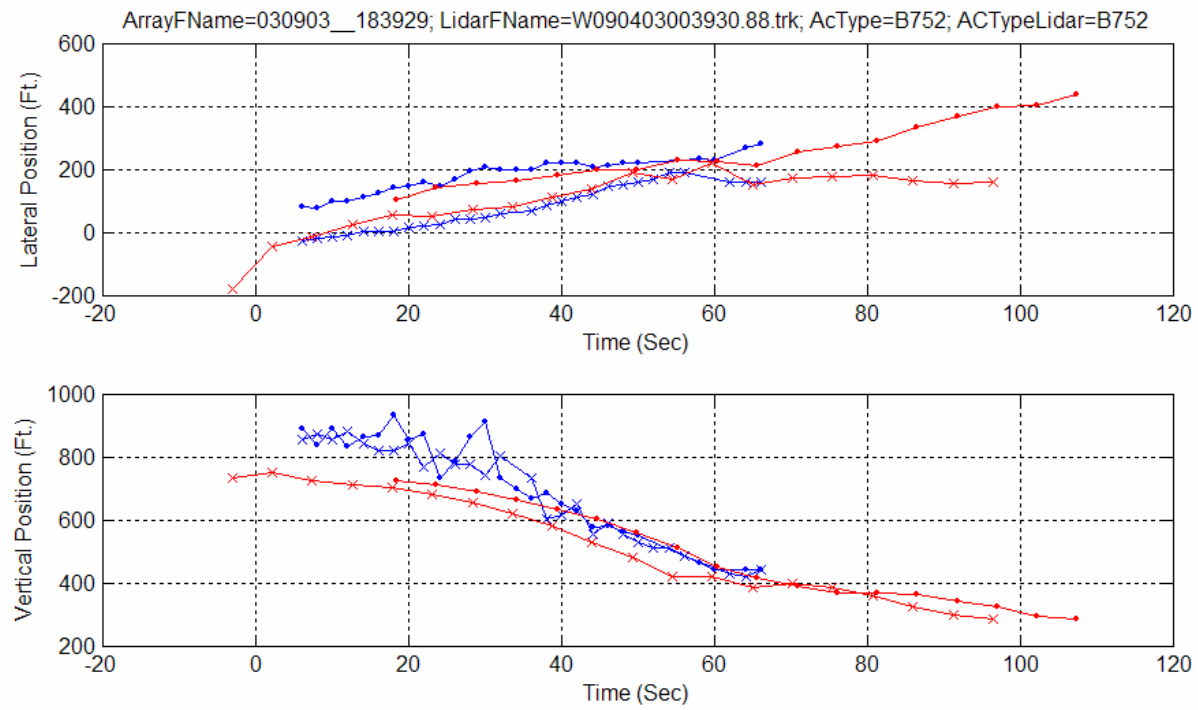

Figure 8. Sample Comparison of the Vortex Trajectory Data from Both the Microphone Array and Pulsed Lidar. The Blue and Red Lines Represent the Microphone Array and pulsed Lidar Tracks, Respectively.

A global comparison in the lateral and vertical positions reported by the phased microphone array and pulsed Lidar is shown in Fig. 9. To facilitate the study, tracks from the two sensors were first interpolated to obtain common times, and comparisons were made only for the overlapping portion of the data. It is also worth noting that the height resolution of the pulsed Lidar is, in principle, better than that of the array, and that the horizontal resolution of the array is better than that of the Lidar. The comparison is, therefore, very complementary. Fig. 9a shows the distribution of the differences between Lidar and array measurements of lateral position. Since the microphone array tracks are not processed through a tracking filter, occasional large difference values are generated due to the array data not being smoothed. Both the median and standard deviation of the difference in the lateral position are superimposed onto the plots. It is seen that a positioning bias exists; the pulsed Lidar, on the average, indicates the wake to be further east than the array reported lateral position. It is uncertain at this time if this difference is purely due to the sensors. Other possibilities that could contribute to bias in the reported position history include site survey errors as well as a nuance in how time is stamped into the pulsed Lidar data between each scan; namely, the incremental wake age in the Lidar data is reported only when the Lidar has completed a scan. Depending on the characteristics of the crosswind in the database that formed the comparison study, a possible bias could have been introduced as the result of this aspect of the Lidar data. These aspects will be examined in the next stage of the various data analyses. Fig. $9 \mathrm{~b}$ shows the distribution of the differences between Lidar and array measurements of vertical position. The occasional large differences observed are again attributed to the unsmoothed nature of the array tracks. Fig. $9 \mathrm{~b}$ indicates that the reported wake position by the array is typically higher than that of the pulsed Lidar. It is believed that the time stamping characteristic of the Lidar data cannot account for the difference observed. The reported difference is therefore most likely related to aspects of the beamforming details. 


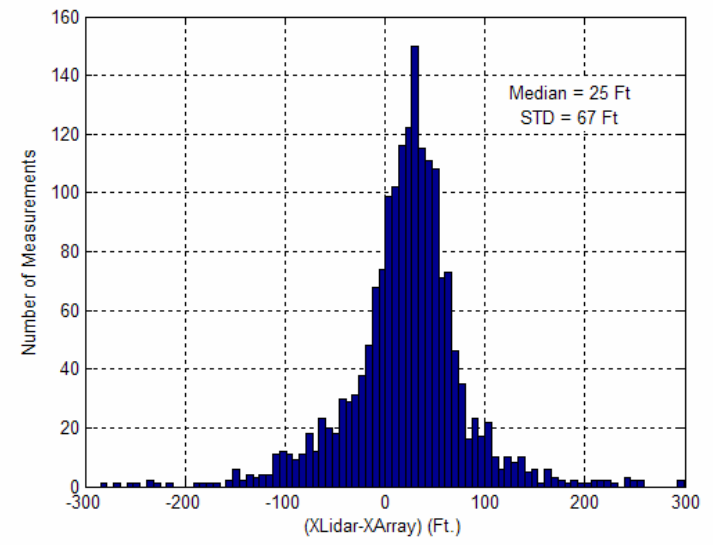

9a. Lateral Position Difference

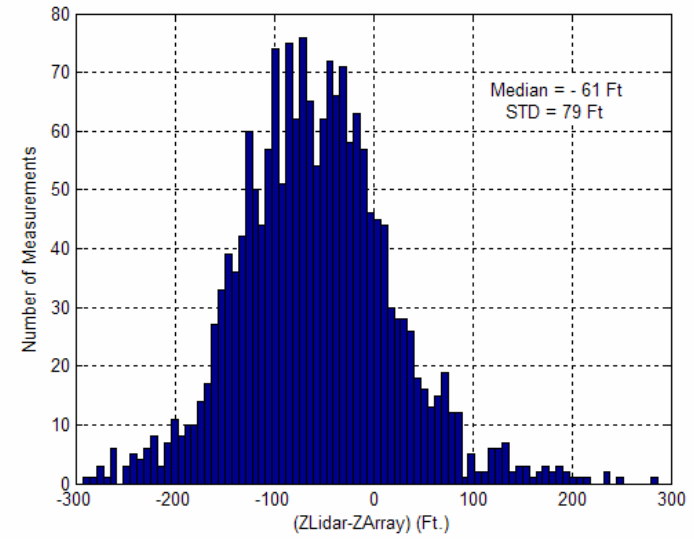

9b. Vertical Position Differences

Figure 9. Distribution of the Tracking Differences.

\section{Correlation of Significant Wake Distortion with Ambient Turbulence}

Since the source localization maps obtained from beamforming on a horizontal plane contain flow visualization like information, an attempt was made to correlate the observed wake dynamics with the most readily available and reliable turbulence measurements to date, which are data from the sonic anemometer. 315 flybys were recorded during the period when the sonic anemometer measurements were available. For this exploratory study, eddy dissipation rate (denoted as EDR, or $\varepsilon$ ) was calculated using the spectral method ${ }^{16}$ from the longitudinal direction of the wind at 30minute intervals. An intermediate averaging was performed on the time series data to determine the incoming flow direction on a five-minute basis. Finally, the present study used a coefficient value of 0.55 in the spectral fitting process. The EDR data are then non-dimensionalized as $\varepsilon^{*}=\left(\varepsilon b_{o}\right)^{1 / 3} / V_{o}$, where $V_{o}$ is the theoretical initial descent velocity of the vortex pair. Following Ref. 15, values of $\varepsilon^{*}$ are then correlated with the non-dimensional time (denoted as $T^{*}$, where the parameters used in the non-dimensionalization follows that of $T$ ) when vortices developed into significantly distorted states. Since the resolution of the array improves with increasing frequency, $200-400 \mathrm{~Hz}$ source localization maps in the horizontal beamforming plane were used primarily for the study, with reference to the $0-200 \mathrm{~Hz}$ counterpart results when needed. Results are compiled for three vortex states and they are shown in Fig. 10.

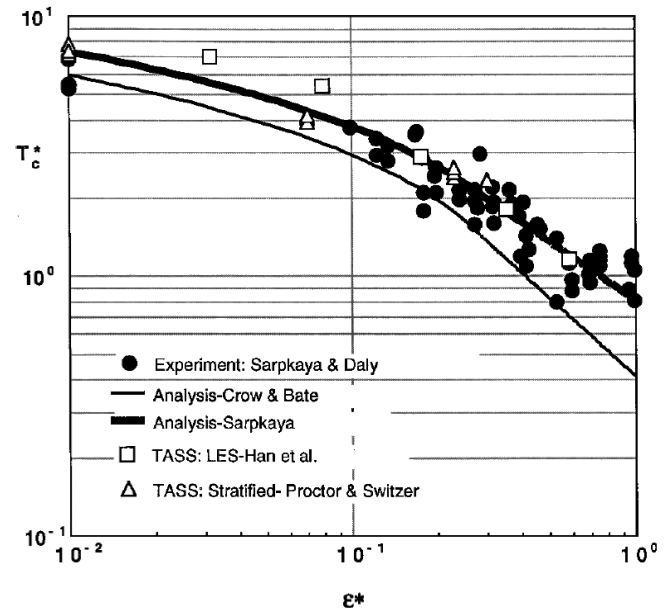

10a. Baseline Graph from Ref. 15

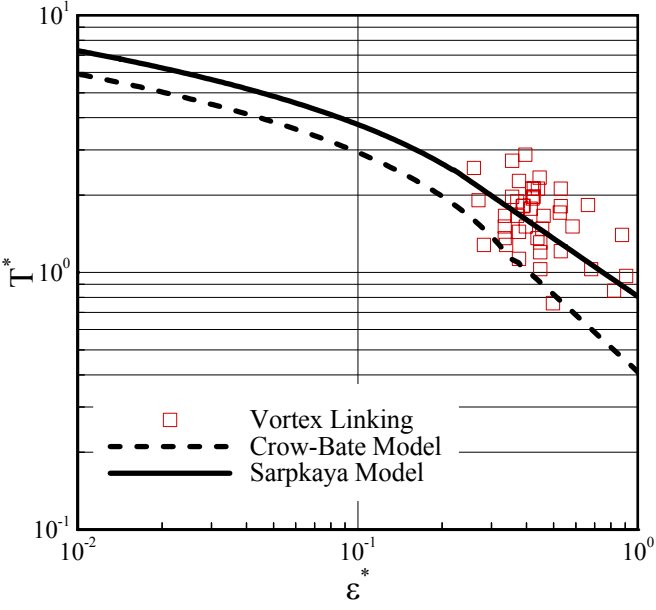

10b. Correlation with Vortex Linking State 


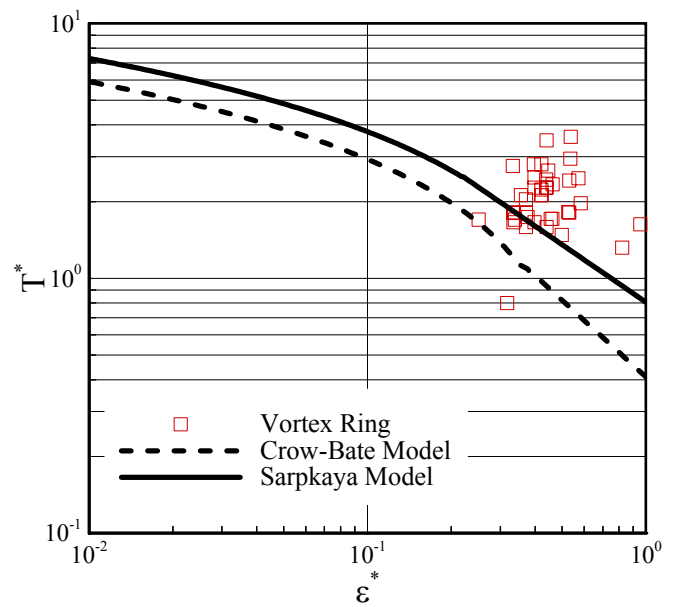

10c. Correlation with Vortex Ring State

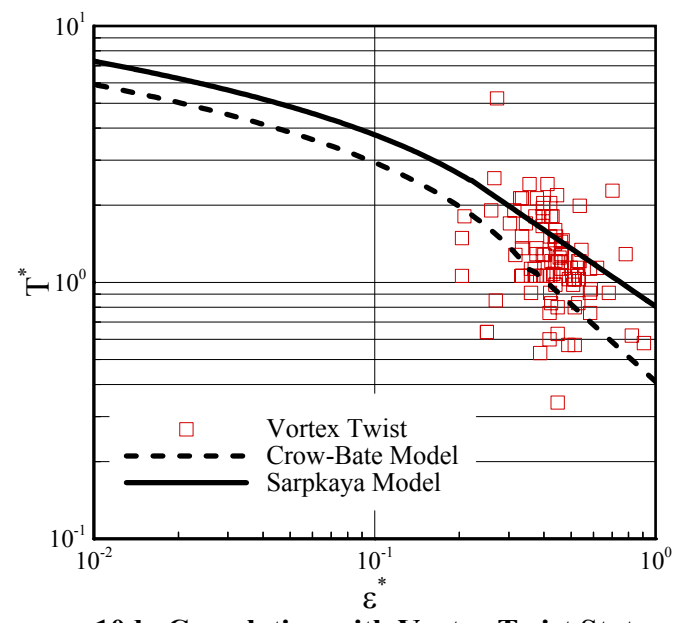

10d. Correlation with Vortex Twist State

Figure 10. Correlation of Vortex Distortion Time Scale to Surface Eddie Dissipation Rate.

Fig. 10a is taken from Ref. 15 and is used to form a baseline comparison with the Denver 2003 beamforming data. Three vortex states are considered, and they are respectively, vortex linking, vortex ring and vortex twisting. Data points from these vortex states are plotted separately to avoid cluttering. Fig. 10b shows the results by considering the vortex linking distortion state and has a reasonable fit to the data points from Fig. 10a as well as with the theoretical trends. The correlation is surprisingly good in light of the fact that 30-minute surface measurements of EDR were used and the lumped effect of shear and stratification is included in the visualization data. Fig. 10c summarizes the vortex ring state results, and it is noted that the points in general are higher than the upper bound theoretical prediction of Sarpkaya $^{15}$ as well as the reference data points of Fig. 10a, as well as those of 10b. These higher points in Fig. 10c represent longer times than those in Fig. 10a or 10b. It is noted that, in vortex evolution, the linking state (Fig. 10b) is followed by the ring state (Fig. 10c), and there can be a significant time lag between vortex linking and the emergence of a fully developed vortex ring. An example of this lag time in beamforming space is exemplified in Fig. 5, thereby can shift the data points higher on the time scale in Fig. 10c.

For completeness, data for wakes to develop into a twisting, contorted appearance is also plotted. From the perspective of horizontal beamforming source localization visualization, it was not always easy to conclusively discriminate between vortex linking and vortex twisting. In general, vortex linking is classified by observing vortices developing into a twisting behavior followed by an intensification of sound at the contact point of the linking process. This description, while helpful, may not always be valid due to the automatic scaling of the color map to include the loudest sound in the beamforming space. It is therefore, conceivable that a portion of the vortex twisting state may in fact be that of vortex linking. A near-future effort will involve a more detailed outlier analysis of the data shown in Fig. 10. It is also envisioned that it could be worthwhile to explore other beamforming and image processing techniques to improve the quality of the images. These additional studies, coupled with finer time resolution of EDR, either from the sonic anemometer or other remote sensing data, could enhance the quality of the dataset shown in Fig. 10.

\section{E. Spectral Characteristics and Correlation with Vortex Strength}

Recall that a set of wake tracks were generated by beamforming in a vertical plane directly above the phased microphone array center. The tracks themselves were then used to guide the focusing point of the array processing to obtain spectra. Using the technique of Ref. 10, background noise was carefully characterized during the spectra generation process. Fig. 11 shows an example of the spectral characterization for a B735 flyby. The aircraft flyover is seen in Fig. 11a at 16 seconds after the start the start of data acquisition. Wake vortex sound is evident throughout the data recording period and is seen to have its frequency content below $400 \mathrm{~Hz}$. Due to the usage of auto-gain in the spectra generation, vortex sound has the apparent loudness equal to that of the aircraft noise at flyover. The dB level shown in the spectrogram is therefore not to be taken as a measure of sound-pressure-level (SPL) relative to human hearing threshold. Instead, Fig. 11a shows the vortex wake sound signal gain relative to the background noise. A representative spectrum, generated at 30 seconds (or 14 seconds after aircraft flyover), is shown in Fig. 11b. The magenta line represents the statistical 90 percent upper confidence limit of the background noise estimated in a 6 second interval $^{10}$ and the combined red-green line represents the spectrum obtained from beamforming on the pre-determined 
vortex trajectory. The red portion of the line is the wake signal unambiguously above the background noise whereas the green portion is still within the statistical bound of the ambient sound. From Fig. 11a, it is seen that spectrum at various times has a strong and consistent component below $100 \mathrm{~Hz}$.

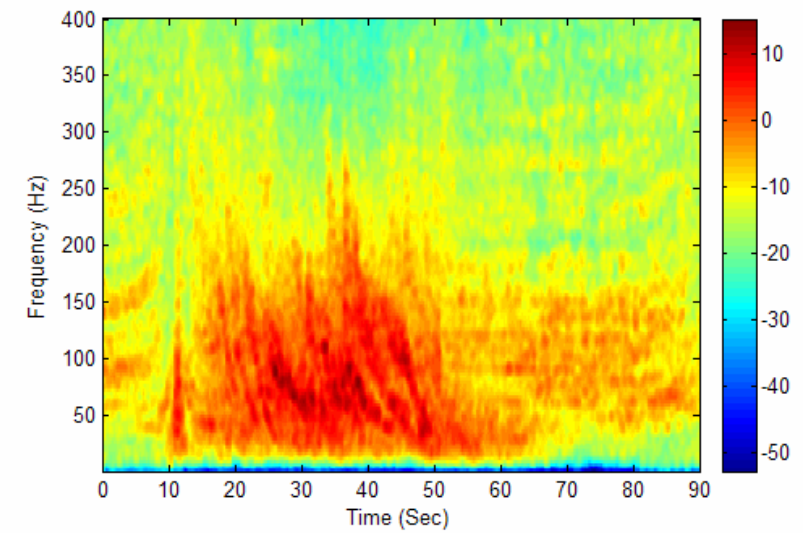

11a. Spectrogram of a B735 Flyby

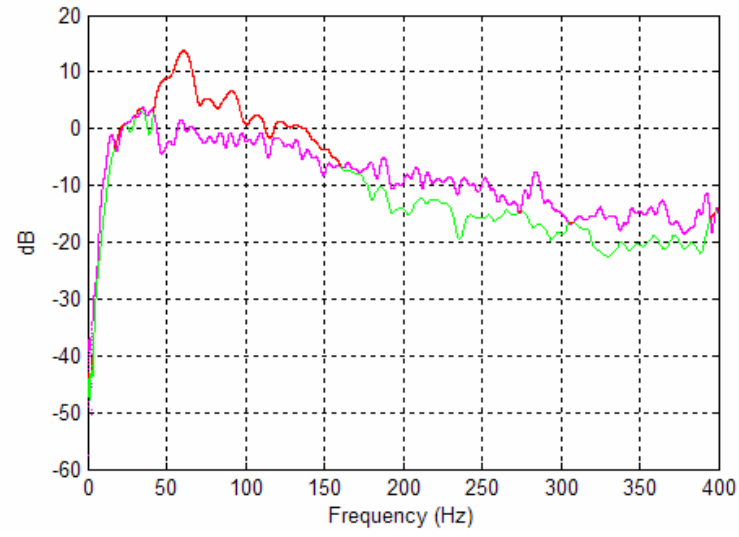

11b. Spectrum Generated at 30 Seconds

Figure 11. Spectral Characteristics of a B735 on September 16, 2003.

The range of the aforementioned frequency "hump" in Fig. 11b in the vortex spectrum can be explained using the Kirchhoff vortex model ${ }^{17-19}$ with the radii of the core restricted to vary between 1.5 to 2 percent of the wingspan. ${ }^{20}$ The Kirchhoff vortex is an elliptical patch of constant vorticity and essentially is a Rankine vortex with an eccentricity. Such a model, therefore, suggests the possibility that the solid body rotation core provides the most consistent source of acoustic radiation in wake vortices, and the frequency range predicted agrees well with the measured spectra. For B737s and B757s, the frequency bound estimated in the aforementioned framework are, 43-76 Hz and 30-54 Hz, respectively. Acoustic power over the range of these frequencies is then integrated as a function of time to characterize the global evolution of wake noise.

The link between aeroacoustics and aerodynamics often follows a power law relationship. ${ }^{21}$ In order to explore whether such a relationship exists between the phased microphone array and pulsed Lidar measurements, the base- 10 logarithm of circulation data from Lidar measurements are presented to facilitate the comparison with the acoustic data expressed in $\mathrm{dB}$. Also for this particular comparison purpose, acoustic data were processed without auto-gain. Figs. 12 and 13 show the comparison between the acoustic power integrated over $43-76 \mathrm{~Hz}$ for B737 aircraft and 30-54 for B757 aircraft, versus the base-10 logarithm of the circulation data in $\mathrm{m}^{2} / \mathrm{s}$. It is noted that with the microphone array and pulsed Lidar data presented in this way, on the average, both exhibit an approximate linear decay over time. There appears to be, therefore, a power law relationship between the two datasets, although the degree of correlation between them is not easily quantifiable at this time.

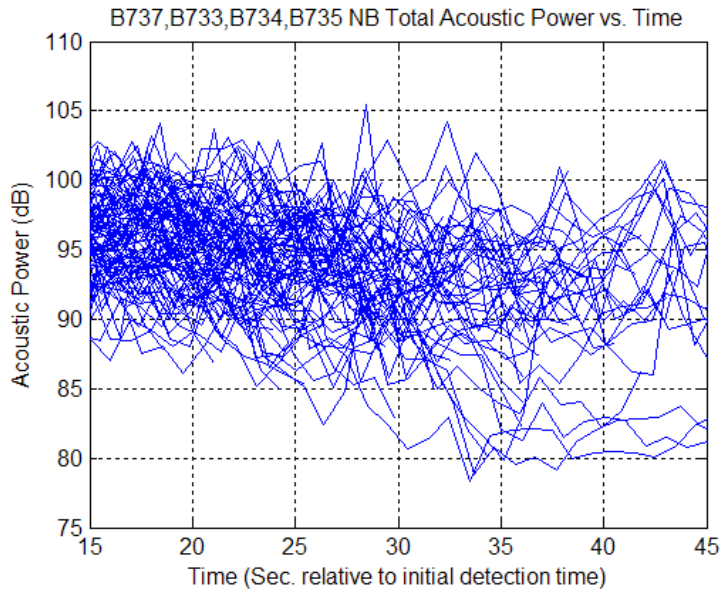

12a. Acoustic Power Evolution of B737 Aircraft Wakes

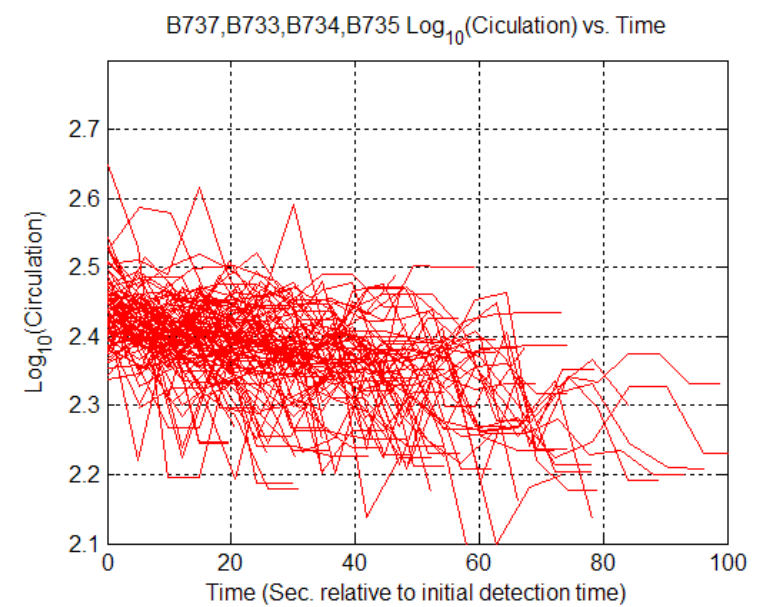

12b. Circulation Evolution of B737 Aircraft Wakes 


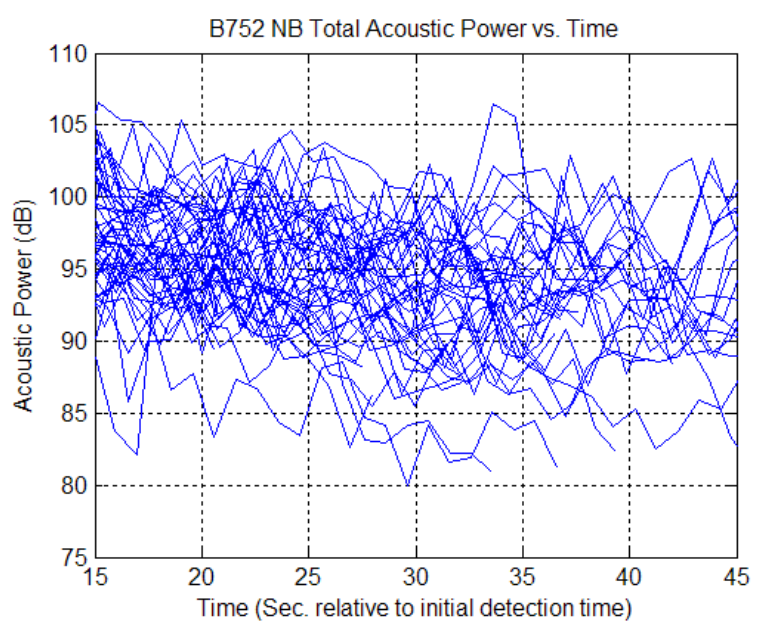

13a. Acoustic Power Evolution of B757 Aircraft Wakes

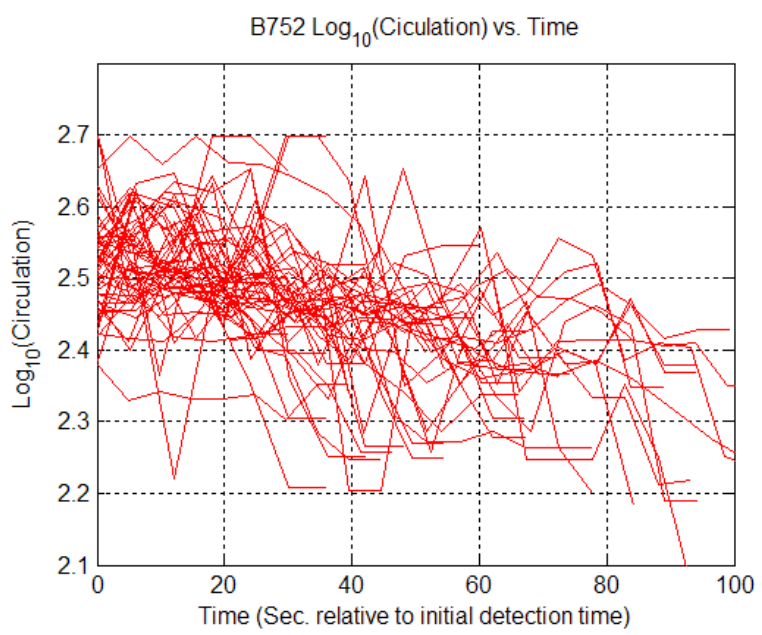

13b. Circulation Evolution of B757 Aircraft Wakes

Figure 13. Correlation Between Narrow Band Acoustic Power and Circulation vs. Time for B757s.

\section{Summary and Closing Remarks}

This paper summarizes recent studies using phased microphone array data to better understand aircraft wake vortex behavior. The overall detection of wake vortex sound in the dataset is better than 80 percent. It is known that each flight generates a wake, but not all wakes appear to generate robust acoustic signatures that can be unambiguously discerned by the array deployed and with the current processing techniques. Nevertheless, through the use of source localization maps, it is demonstrated that the phased microphone array deployed in the Denver 2003 study can provide visualization of wake vortices in an operational environment. Utilizing this special capability of the sensor, research issues such as estimating the initial vortex separation distance, correlation of the time scale for wakes to develop into a highly contorted state with ambient turbulence and comparison of microphone inferred wake tracks with pulsed Lidar data are examined. Using a phased microphone array, which incorporates a very different physical principle from other conventional wake vortex sensors, the paper confirms that ambient turbulence has a dominant role in OGE wake evolution. It is also noteworthy that the current paper substantially enlarged the amount of flight data available for this particular aspect of vortex dynamics. An example was also given to illustrate how the microphone array data can be combined with the pulsed Lidar data to provide a more complete understanding of the evolution of an aircraft vortex wake.

Spectrally, vortices in OGE generate broadband sound with most of the energy residing below $400 \mathrm{~Hz}$. However, a portion of the spectrum below $100 \mathrm{~Hz}$ appears to be consistently above ambient background, whose frequency range can be obtained using a simple modified Kirchhoff vortex model. Finally, preliminary examination of the acoustic power history over the aforementioned band of frequencies vs. Lidar circulation measurements suggests that a power law relationship between the two datasets may exist, although its degree of correlation is not quantified at this time.

\section{Acknowledgments}

The authors thank Wayne H. Bryant of NASA Langley Research Center and Michael Geyer of USDOT Volpe Center for their encouragement and support. We also gratefully acknowledge Robert P. Dougherty of OptiNav, Inc., for valuable discussions as well as providing the $0-200 \mathrm{~Hz}$ beamforming results. We sincerely thank Titan Corporation, Microstar Laboratories, Inc. and Denver International Airport authority for the logistic support. We also thank James N. Hallock for proofreading the manuscript. Finally, we acknowledge the fantastic assistance from Denver air traffic controllers for directing as many arrivals to Runway $16 \mathrm{~L}$ during the test period as conditions permitted.

\section{References}

${ }^{1}$ Burnham, D., Gorstein, M., Hallock, J. N., Kodis, R., Sullivan, T., and McWilliams, I. G., “Aircraft Wake Vortex Sensing Systems,” DOT-TSC-FAA-72-13, June 1971, DOT Transportation Systems Center, Cambridge, MA. 
${ }^{2}$ Hallock, J. N., and Eberle, W. R., (ed.), "Aircraft Wake Vortices: A State-of-the-Art Review of the United states R\&D Program," FAA-RD-77-23, February 1977, DOT Transportation Systems Center, Cambridge, MA.

${ }^{3}$ Cotton, W. and William, R., "PROJECT SOCRATES: A New Sensor Technology for Enhancement of Aviation Safety and Capacity," The 2002 FAA Airport Technology Conference, Atlantic City, NJ, May 5 - 8, 2002.

${ }^{4}$ Michel, U., and Böhning, P., "Investigation of Aircraft Wake Vortices with Phased Microphone Arrays," AIAA Paper 20022501, 8th AIAA/CEAS Aeroacoustics Conference, Breckenridge, CO, June 2002.

${ }^{5}$ Wang, F. Y., Wassaf, H., Dougherty, R. P., Clark, K., Gulsrud, A., Fenichel, N., and Bryant, W. H., "Passive Wake Acoustics Measurements at Denver International Airport," 4th NASA Integrated CNS Conference and Workshop, Fairfax, VA, April 2004.

${ }^{6}$ Dougherty, R. P., Wang, F. Y., Booth, E. R., Watts, M. E., Fenichel, N., and D'Errico, R. E., “Aircraft Wake Vortex Measurements at Denver International Airport," AIAA Paper 2004-2880, 10th AIAA/CEAS Aeroacoustics Conference, Manchester, UK, May 2004.

${ }^{7}$ Dougherty, R. P., "Extensions of DAMAS and Benefits and Limitations of Deconvolution in Beamforming," AIAA Paper 20052961, 11th AIAA/CEAS Aeroacoustics Conference, Monterey, CA, May 2005.

${ }^{8}$ Booth, E. R., Jr., and Humphreys, W. M., Jr., "Tracking and Characterization of Aircraft Wakes Using Acoustic and Lidar Measurements," AIAA Paper 2005-2964, 11th AIAA/CEAS Aeroacoustics Conference, Monterey, CA, May 2005.

${ }^{9}$ Fine, N. E., and Kring, D. C., "Opto-Acoustic Tracking of Aircraft Wake Vortices," AIAA Paper 2005-2965, 11th AIAA/CEAS Aeroacoustics Conference, Monterey, CA, May 2005.

${ }^{10}$ Wassaf, H. S., Ibe, O. C., Dougherty, R. P., and Zhang, Y., "Acoustical Spectral Analysis of a Wake Vortex Cross-Section Using Microphone-Arrays," The Journal of the Acoustical Society of America, Vol. 117, Issue 4, April 2005, p. 2546.

${ }^{11}$ Hannon, S. M., Phillips, M. W., Thompson, J. A., and Henderson, S. W., "Pulsed Coherent Lidar Wake Vortex Detection, Tracking and Strength Estimate in Support of AVOSS," NASA First Wake Vortex Dynamic Spacing Workshop - NASA CP-97-

206235, 1997, NASA Langley Research Center, Hampton, VA.

${ }^{12}$ Hannon, S. M., "Pulsed Doppler Lidar for Terminal Area Monitoring of Wind and Wake Hazards," American Meteorological Society 11th Conference on Aviation, Range, and Aerospace Meteorology, Hyannis, MA, October 2004.

${ }^{13}$ Crow, S. C., "Stability Theory for a Pair of Trailing Vortices," AIAA Journal, Vol. 8, No. 12, 1970, pp. $2172-2179$.

${ }^{14}$ Crow, S. C., and Bate, E. R., "Lifespan of Trailing Vortices in a Turbulent Atmosphere," Journal of Aircraft, Vol. 13, No. 7, 1976, pp. 476-482.

${ }^{15}$ Sarpkaya, T., Robins, R. E., and Delisi, D. P., "Wake-Vortex Eddy-Dissipation Model Predictions Compared with Observations," Journal of Aircraft, 38, 2001, pp. 687-692.

${ }^{16}$ Britt, C. L., Kelly, C. W., and Nguyen, D. P. C., "Algorithms for Generation of Wind Turbulence Profiles from Pulsed Lidar Measurements,” RTI Technical Report RTI/8348/010-01F, March 2003, Research Triangle Institute, NC.

${ }^{17}$ Howe, M. S., Theory of Vortex Sound, Cambridge University Press, Cambridge, UK, 2002.

${ }^{18}$ Müller, B., "On Sound Generation by the Kirchhoff Vortex," Uppsala University Department of Scientific Computing Report No. 209-1998, October 1998, Uppsala University, Sweden.

${ }^{19}$ Hardin, J. C., and Wang, F. Y., "Sound Generation by Aircraft Wake Vortices,” NASA CR-2003-212674, December 2003, NASA Langley Research Center, VA.

${ }^{20}$ Delisi, D. P., Greene, G. C., Robins, R. E., Vicroy, D. C., and Wang, F. Y., “Aircraft Wake Vortex Core Size Measurements," AIAA Paper 2003-3811, 21st Applied Aerodynamics Conference, Orlando, FL, June 2003.

${ }^{21}$ Lilley, G. M., “A Quest for Quiet Commercial Passenger Transport Aircraft for Take-off and Landing,” AIAA Paper 20042922, 10th AIAA/CEAS Aeroacoustics Conference, Manchester, UK, May 2004. 